\title{
Traversable wormholes in five-dimensional Lovelock theory
}

\author{
Gaston Giribet, Emilio Rubín de Celis, and Claudio Simeone \\ Physics Department, University of Buenos Aires and IFIBA-CONICET Ciudad Universitaria, \\ pabellón 1, 1428 Buenos Aires, Argentina
}

(Received 11 June 2019; published 8 August 2019)

\begin{abstract}
In general relativity, traversable wormholes are possible provided they do not represent shortcuts in the spacetime. Einstein equations, together with the achronal averaged null energy condition, demand to take longer for an observer to go through the wormhole than through the ambient space. This forbids wormholes from connecting two distant regions in the space. The situation is different when higher-curvature corrections are considered. Here, we construct a traversable wormhole solution connecting two asymptotically flat regions, otherwise disconnected. This geometry is an electrovacuum solution to the Lovelock theory of gravity coupled to an Abelian gauge field. The electric flux suffices to support the wormhole throat and to stabilize the solution. In fact, we show that, in contrast to other wormhole solutions previously found in this theory, the one constructed here turns out to be stable under scalar perturbations. We also consider wormholes in anti-de Sitter (AdS). We present a protection argument showing that, while stable traversable wormholes connecting two asymptotically locally $\mathrm{AdS}_{5}$ spaces do exist in the higher-curvature theory, the region of the parameter space where such solutions are admitted lies outside the causality bounds coming from AdS/CFT.
\end{abstract}

DOI: 10.1103/PhysRevD.100.044011

\section{INTRODUCTION}

Wormholes are one of the most fascinating solutions of gravitational field equations. Originally conceived as a hypothesis on the structure of matter in classical physics [1], wormholes have served as illustrative examples on how abstruse the topology of spacetime can be [2], allowing us to investigate to what extent causality, locality, and energy conditions are interrelated [3]. These geometries have also provided a particularly rich source of inspiration for science fiction literature. The science fiction wormholes, however, are radically different from those seriously considered in theoretical physics; the main difference being their inviability at macroscopic scales. The existence of wormholes demands the violation of certain energy conditions, which is only possible in quantum physics.

In the past few years, the interest on wormhole geometries have been renewed. Both traversable and nontraversable wormhole geometries were recently considered in relation to quantum gravity and notably to holography [4-13]. In [13], an explicit example of a metastable, traversable wormhole was constructed. ${ }^{1}$ The solution

\footnotetext{
${ }^{1}$ There have been other interesting papers recently on wormhole geometries; see for instance [14-17] and references therein and thereof.

Published by the American Physical Society under the terms of the Creative Commons Attribution 4.0 International license. Further distribution of this work must maintain attribution to the author(s) and the published article's title, journal citation, and DOI. Funded by SCOAP .
}

describes a pair of extremal magnetically charged black holes connected by a long throat, in such a way that it takes longer for an observer to go through the wormhole than through the ambient space, as demanded by the inalienable achronal averaged null energy condition [18]. Einstein equations, once such an energy condition on the matter fields was imposed, do not allow for wormholes with a short throat. In particular, wormholes connecting two distinct asymptotically flat regions are excluded. The situation is different when higher-curvature corrections to the gravitational action are taken into account. In that case, the higher-degree ${ }^{2}$ terms can effectively act as the exotic matter contribution needed for such wormholes to exist.

Here, we will explicitly show that higher-curvature models in five dimensions do allow for stable wormholes that connect two asymptotically flat-or asymptotically anti-de Sitter (AdS) - regions without introducing extra exotic matter. As a working example, we will consider the Einstein-Maxwell theory supplemented with the quadratic Gauss-Bonnet higher-curvature terms; namely

$$
\begin{aligned}
S= & \frac{1}{16 \pi} \int d^{5} x \sqrt{-g}\left(R-2 \Lambda-F_{\mu \nu} F^{\mu \nu}\right. \\
& \left.+\alpha\left(R_{\mu \nu \rho \sigma} R^{\mu \nu \rho \sigma}-4 R_{\mu \nu} R^{\mu \nu}+R^{2}\right)\right)+B,
\end{aligned}
$$

\footnotetext{
${ }^{2}$ In dimensions greater than four, higher-curvature terms do not necessarily yield higher-order terms in the field equations. They can well lead to second-order field equations that are nonlinear in the second derivative of the fields.
} 
where $B$ stands for the boundary term that renders the variational problem well posed. This theory is the favorite model to study the effects of higher-order terms in the context of $\mathrm{AdS}_{5} / \mathrm{CFT}_{4}$ correspondence $[19,20]$, the reason being that it is analytically solvable in many physically attractive scenarios such as AdS black holes. Besides, action (1) can be motivated from string theory: it resembles the $\alpha^{\prime}$-corrected low energy effective action of heterotic string theory, and it also appears in Calabi-Yau compactification of $\mathrm{M}$ theory to five dimensions. ${ }^{3}$ The specific combination of quadratic terms in (1) is the only one that yields second-order field equations [24,25]. As a consequence, the theory is free of Ostrogradsky instabilities. It also results as free of ghosts around its perturbative maximally symmetric vacuum [26]. The theory, however, exhibits some causality issues [27] and other notorious features [28]. In [27], the causality constraints of highercurvature models were studied, and it was shown in particular that a theory such as (1) has to be supplemented with massive higher-spin fields in order to be free of causality problems. Causal structure of Einstein-GaussBonnet (EGB) theory has also been studied in $[29,30]$, where different notions closely connected to causality are studied in detail, such as the relation between Killing horizons and characteristic hypersurfaces, hyperbolicity in the near horizon regions.

Theory (1) is a particular case of the so-called Lovelock theory of gravity [31], which is a natural generalization of Einstein theory to higher dimensions [25]. It is defined as the dimensional extension of a topological invariant that, in virtue of the Chern-Weil generalization of the GaussBonnet theorem, computes the Euler characteristic of a 4-manifold. In such a theory, we will construct traversable wormhole geometries supported by a Maxwell field without introducing exotic matter and, as a consequence, satisfying the energy conditions. Our wormhole solution connects two different asymptotically flat or AdS regions. In other words, it represents a "short" wormhole. The impediments that one finds when trying to construct such a solution in Einstein theory are circumvented here due to the presence of higher-curvature terms, which suffice to support the throat. This implies, in particular, that the wormhole will be microscopic, i.e., with a throat of the size $\sqrt{\alpha}$. The geometry still represents an exact solution to (1) which, in contrast to other solutions found previously [32-34], is stable under $S$-perturbations. It is the presence of the $U(1)$ charge that stabilizes the wormhole and makes the asymptotically flat solution possible.

The fact that the wormhole throat is of order $\sqrt{\alpha}$ raises an immediate objection: At that scale, terms that are higher in

\footnotetext{
${ }^{3}$ The first higher-curvature correction of $\mathrm{M}$ theory in 11 dimensions is a quartic term, $R^{4}[21,22]$. When compactifying the theory on a six-dimensional Calabi-Yau, the effective fivedimensional theory exhibits $R^{2}$ terms as those in (1); see for instance Eqs. (2.6)-(2.9) in [21]; see also Eq. (1) in [23].
}

the curvature would contribute, and so they cannot be neglected. If we think of (1) as the truncation of an ultraviolet complete theory, the effective theory would break down at that scale. In addition, we will see that the geometric construction of the higher-curvature wormholes requires one to consider certain internal patches of the spacetime solutions to (1) that are nonperturbative, in the sense of having $S_{\text {on-shell }} \sim 1 / \alpha$-these solutions are somehow analogous to the self-accelerated solutions of higherderivative models. However, one can in principle answer these objections in the following way: Despite that highercurvature terms cannot be neglected at length scales $\sqrt{\alpha}$, one has the expectation that the qualitative features introduced by the terms quadratic in the curvature will not be drastically affected by the introduction of, say, quartic or higher terms. More precisely, one can imagine a window in the parameter space such that the wormhole throat, although microscopic, is still larger than the length at which quartic terms become relevant. We will adopt here a pragmatic point of view: As in other works in which highercurvature actions such as (1) are considered-notably in the context of $\mathrm{AdS}_{5} / \mathrm{CFT}_{4}$ - and even when such actions seem to violate the logic of effective field theory and require finetuning, we will take into account that holography does not exclude such models, and so they might in principle be considered [35].

We should probably add that wormhole solutions in fivedimensional EGB theory (1) have already been discussed in the literature [36-40]. Our solutions, however, differ from those considered before in many aspects. For example, the main differences between the wormhole solutions considered in [36-38] and ours are the following: First, the solutions considered therein only exist at the so-called Chern-Simons point $\Lambda \alpha=-3 / 4$ [41], while our solutions exist for a continuous range of the parameter $\alpha$ after the cosmological constant has been fixed. Second, the base manifold in the solution of [36-38] is different from those of our examples: while we consider three-dimensional constant curvature manifolds for the constant-time, constant-radius foliation of the geometry, the base manifold of the solution considered in [36-38] is the product of a two-dimensional locally hyperbolic space $H_{2} / \Gamma$ and a circle $S^{1}$. A third difference is that our solutions are constructed by gluing together different patches, all of them corresponding to different values of $\xi$ in the spherically symmetric solution (3) below. In contrast, the solution of [36-38] is given by a smooth $C^{\infty}$ single manifold-at the Chern-Simons point, the different effective cosmological constants of the higher-curvature Lovelock theory coincide. Our solutions also differ from the class of solutions studied in [39]. There, the authors find some conditions and no-go propositions for the existence of wormhole-type solutions in EGB theory coupled to matter. Those results are certainly consistent with our finding: For instance, in proposition 2 of [39], the point is made that the null energy condition has to be 
violated if the wormhole's throat is embedded in the so-called general relativity (GR) branch of EGB theory [which corresponds to the choice $\xi=1$ in (3) below]. Our solution does obey the null energy condition on the throat precisely because its throat is embedded in the opposite branch [the branch $\xi=-1$ of (3) below]. Related to this, it is important to mention that the solutions we consider here also differ from those considered in Ref. [32], where non-GR branches were also considered: In contrast to those in [32], the solutions we construct here have attached an asymptotic region with an arbitrary small effective cosmological constant. In addition, the presence of the electric flux renders our solutions stable under scalar perturbations, unlike those in [32]; this also makes our solutions different from those analyzed, for instance, in [40].

This paper is organized as follows: In Sec. II, we will consider the spherically symmetric, static solutions to the higher-curvature theory coupled to a Maxwell field. These solutions will be the building blocks of our geometry, while the blinder agent will be the junction conditions derived from the boundary term $B$ in (1). These conditions are the generalization of the Israel junction conditions of general relativity. We will discuss this also in Sec. II. In Sec. III, we will construct the wormhole by assembling different patches of the spacetime. We will do this without resorting to exotic matter. The electric field and the higher-curvature terms will suffice to support the wormhole throat. In Sec. IV, we will study the stability conditions for our wormhole solution. We will show that, in certain regions of the parameter space, the solution turns out to be stable under scalar perturbations. In Sec. V, we will generalize the solution to the case of asymptotically $\mathrm{AdS}_{5}$ wormholes, and we will make some comments in relation to AdS/CFT correspondence.

\section{HIGHER-CURVATURE GRAVITY}

Let us begin by considering the black hole solutions of the theory defined by $(1)$, namely $[42,43]$

$d s^{2}=g_{\mu \nu} d x^{\mu} d x^{\nu}=-f(r) d t^{2}+f^{-1}(r) d r^{2}+r^{2} d \Omega^{2}$,

where $d \Omega^{2}$ is the metric on a 3-space of constant curvature $k=0, \pm 1$, and where the function $f$ is given by

$f(r)=k+\frac{r^{2}}{4 \alpha}\left(1+\xi \sqrt{1+\frac{16 \alpha M}{r^{4}}-\frac{8 \alpha Q^{2}}{3 r^{6}}+\frac{4 \alpha \Lambda}{3}}\right)$,

where $\xi= \pm 1$. Here, $t \in \mathbb{R}, r \in \mathbb{R}_{\geq 0}$. The solution with $\xi=-1, k=1$, and $\Lambda=0$, in the large $r$ limit, tends to the five-dimensional Reissner-Nordström solution of the Einstein-Maxwell theory. The nonzero components of the spherically symmetric electromagnetic field are $F^{t r}=Q / r^{3}$, with $Q$ being the electric charge. We consider ${ }^{4}$

\footnotetext{
${ }^{4}$ This is the sign compatible with string theory.
}

$\alpha>0$ and, mainly, asymptotically flat spacetimes which correspond to $\xi=-1$ solutions and vanishing cosmological constants. These are $k=1$ spherical geometries with a branch singularity at $r=r_{S}$, shielded by two horizons for charges $|Q|<Q_{c}=\sqrt{3}|M-\alpha|$, with $M$ the ArnowittDeser-Misner mass. If $|Q|=Q_{c}$, there is one horizon only, and for $|Q|>Q_{c}$ there is a naked singularity at the radius where the radicant in (3) vanishes; i.e., at the surface $r=r_{S}$, where $r_{S}$ is the largest real solution to the equation $r_{S}^{6}(1+4 \alpha \Lambda / 3)+r_{S}^{2} 16 \alpha M-8 \alpha Q^{2} / 3=0$. The outer horizon for $|Q|<Q_{c}$ is at $r=r_{H}$, where

$$
r_{H}^{2}=\frac{Q_{c}}{\sqrt{3}}+\sqrt{\frac{Q_{c}^{2}-Q^{2}}{3}} .
$$

As said in the Introduction, our wormhole will be constructed by joining together different patches of the geometry (2). As junctures, we consider timelike shells which separates two bulk geometries with some $f_{-}(r)$ and $f_{+}(r)$ as in (3). These shells are described by radial coordinate $r=\rho(\tau)$ and time coordinates $t_{\mp}=T_{\mp}(\tau)$, where the parameter $\tau$ is the proper time on the shell [44]. The induced metric $d h^{2}=-d \tau^{2}+\rho^{2}(\tau) d \Omega^{2}$ is the same from both sides, and then $f_{\mp} \dot{T}_{\mp}^{2}-f_{\mp}^{-1} \dot{\rho}^{2}=1$ at the shell. The induced stress tensor $S_{i j}$ at the hypersurface is given by

$-8 \pi S_{i j}=\left.\left(K_{i j}-K h_{i j}+2 \alpha\left(3 J_{i j}-J h_{i j}+2 P_{i k l j} K^{k l}\right)\right)\right|_{-} ^{+}$,

where Latin indices label the coordinates on the joining surface of induced metric $h_{i j},\left.\mathcal{F}\right|_{-} ^{+} \equiv \mathcal{F}_{+}-\eta \mathcal{F}_{-}$, with $\eta=$ \pm 1 depending on the relative orientation in both sides of the shell, $K_{i j}$ is the extrinsic curvature tensor over the hypersurface at each side, and the divergence-free parts of the Riemann tensor $P_{i j k l}$ and the tensor $J_{i j}$ are defined as

$$
\begin{aligned}
P_{i j k l}= & R_{i j k l}+R_{j k} h_{l i}-R_{j l} h_{k i}-R_{i k} h_{l j}+R_{i l} h_{k j} \\
& +\frac{1}{2} R\left(h_{i k} h_{l j}-h_{i l} h_{k j}\right),
\end{aligned}
$$

$J_{i j}=\frac{1}{3}\left(2 K K_{i k} K_{j}^{k}+K_{k l} K^{k l} K_{i j}-2 K_{i k} K^{k l} K_{l j}-K^{2} K_{i j}\right)$.

Junction conditions (5), which follows from the boundary term $B$ in (1), are the generalization of the Israel conditions to the case in which higher-curvature terms are included [45].

\section{WORMHOLE SOLUTION}

We will construct electrovacuum wormhole solutions to (1) with two asymptotic regions. The spacetime is symmetric across the throat, and outside the mouth of the wormhole the solution corresponds to a charged, static black hole geometry. We mainly focus on the case of 
asymptotically flat configurations, but we keep track of a nonvanishing cosmological constant to later extend it to the case of wormholes in $\mathrm{AdS}_{5}$ and make some comments in relation to AdS/CFT.

Our solution is constructed with four bulk pieces, four distinct patches joined by three 4-surfaces. Each piece is a region obtained from metric (2) by removing part of the geometry on one side of some codimension-one hypersurface defined at a fixed radial coordinate. The four bulk regions are pasted to construct the geodesically complete manifold $\mathcal{M}$ describing our traversable wormhole. We denote the four regions as left exterior $\mathcal{M}_{L}^{e}$, left interior $\mathcal{M}_{L}^{i}$, right interior $\mathcal{M}_{R}^{i}$, and right exterior $\mathcal{M}_{R}^{e}$, with their respective coordinates $x_{L, R}^{\alpha}$ for the left $(L)$ and right $(R)$ bulks. Explicitly, they are

$$
\begin{aligned}
& \mathcal{M}_{L}^{e}=\left\{x_{L}^{\alpha} / r_{L} \geq b\right\}, \quad \mathcal{M}_{L}^{i}=\left\{x_{L}^{\alpha} / b \geq r_{L} \geq a\right\}, \\
& \mathcal{M}_{R}^{i}=\left\{x_{R}^{\alpha} / a \leq r_{R} \leq b\right\}, \quad \mathcal{M}_{R}^{e}=\left\{x_{R}^{\alpha} / b \leq r_{R}\right\},
\end{aligned}
$$

and the complete manifold is $\mathcal{M}=\mathcal{M}_{L}^{e} \cup \mathcal{M}_{L}^{i} \cup \mathcal{M}_{R}^{i} \cup \mathcal{M}_{R}^{e}$. Each external region $\mathcal{M}_{L, R}^{e}$ is joined to its corresponding inner region $\mathcal{M}_{L, R}^{i}$ at the hypersurface of a bubble $\Sigma_{L, R}^{b}$. In other words, $\Sigma_{L, R}^{b}=\mathcal{M}_{L, R}^{i} \cap \mathcal{M}_{L, R}^{e}$. The left bubble is placed at $r_{L}=b$ and the right bubble is placed symmetrically at $r_{R}=b$. Inner regions are also glued to each other at the throat located at $r_{L}=a=r_{R}$, with $a<b$; i.e., $\Sigma^{a}=\mathcal{M}_{L}^{i} \cap \mathcal{M}_{R}^{i}$. This is depicted in Fig. 1. The junction hypersurfaces are described as $\Sigma_{L}^{b}=\partial \mathcal{M}_{L}^{e}=\left.\partial \mathcal{M}_{L}^{i}\right|_{b}$ and $\Sigma_{R}^{b}=\partial \mathcal{M}_{R}^{e}=\left.\partial \mathcal{M}_{R}^{i}\right|_{b}$ which define bubbles, and $\Sigma^{a}=$ $\left.\partial \mathcal{M}_{L}^{i}\right|_{a}=\left.\partial \mathcal{M}_{R}^{i}\right|_{a}$ which corresponds to the wormhole throat.

The metric function is $f_{i}\left(r_{L, R}\right)$ for interior geometries and $f_{e}\left(r_{L, R}\right)$ for the exteriors, with mass parameters $M_{i}=M_{L i}=M_{R i}$ and $M=M_{L e}=M_{\mathrm{Re}}$, respectively. The exterior metrics must belong to the general relativity branch $\xi_{e}=-1$ and have a vanishing cosmological constant in order to have flat asymptotics and, consequently,

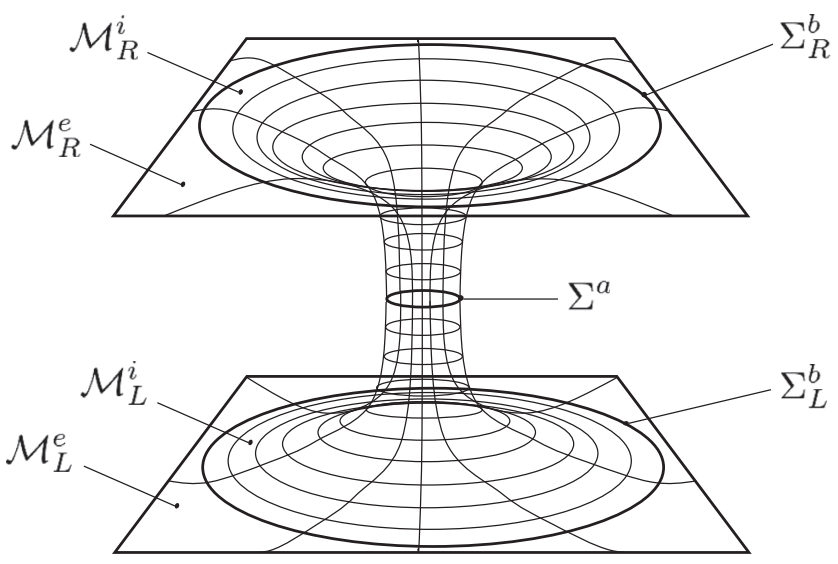

FIG. 1. Scheme of the wormhole geometry. we set $\Lambda=0$. The hypersurface at the throat determines that the inner regions must both be of the $\xi_{i}=+1$ branch to construct the symmetric wormhole. ${ }^{5}$ The configuration implies a throat whose radial coordinate is greater than the branch singularity of the interior metrics, $a>r_{S_{i}}$, while bubbles' radii must be greater than the would-be horizons of the external black hole metrics or their singularity surfaces; $b>\operatorname{Max}\left\{r_{S_{e}}, r_{H}\right\}$.

We are looking for solutions without matter sources, so that the construction requires zero energy density and pressure at the bubbles and throat, implying vanishing induced stress tensor at the shells. A Gauss law integration of Maxwell equations across the noncharged surfaces of the shells, $\oint F_{\mu \nu} d X^{\mu \nu}=0$ with $d X^{\mu \nu}=r^{[\mu} t^{\nu]} d \Omega$, gives the continuity of the electromagnetic field in the temporal $t^{\mu}$ and radial $r^{\nu}$ directions, as

$$
\left.\left(\eta F_{\mu \nu} t^{\mu} r^{\nu}\right)\right|_{-} ^{+}=0
$$

where + and - indicate the bulks at each side of the shell, with the orientation factor $\eta$ of each bulk region as $\eta=+1$ for radial coordinates pointing from left to right in the wormhole manifold, or $\eta=-1$ if the radial coordinates point in the opposite direction. The latter condition can be given in an orthonormal frame as $\left(\eta F_{\hat{t} \hat{r}}\right)_{+}=\left(\eta F_{\hat{t} \hat{r}}\right)_{-}$or, using the metric function $f=-g_{t t}=g_{r r}^{-1}$, directly as $\left(\eta F_{t r}\right)_{+}=\left(\eta F_{t r}\right)_{-}$. From the continuity of the electromagnetic field across the shells, it is seen that the charges are $Q=Q_{R}=-Q_{L}$.

Junction conditions for a generic timelike hypersurface separating two bulk regions with metric functions $f_{-}$and $f_{+}$, located at radial coordinate $r=\tilde{\rho} \equiv \rho(\tau)$, establish a diagonal stress tensor (5) at the shell with components given explicitly by

$$
\begin{aligned}
S_{\tau}^{\tau}= & \left.\eta \frac{\alpha}{2 \pi \tilde{\rho}^{3}} \sqrt{f(\tilde{\rho})+\dot{\tilde{\rho}}^{2}}\left[3\left(k+\frac{\tilde{\rho}^{2}}{4 \alpha}\right)-f(\tilde{\rho})+2 \dot{\tilde{\rho}}^{2}\right]\right|_{-} ^{+}, \\
S_{\theta}^{\theta}= & S_{\phi}^{\phi}=S_{\chi}^{\chi}=\eta \frac{1}{4 \pi \tilde{\rho} \sqrt{f(\tilde{\rho})+\dot{\tilde{\rho}}^{2}}} \\
& \times\left[\frac{\alpha}{\tilde{\rho}}\left(k+\frac{\tilde{\rho}^{2}}{4 \alpha}-f(\tilde{\rho})\right) f^{\prime}(\tilde{\rho})+f(\tilde{\rho})+\dot{\tilde{\rho}}^{2}\right. \\
& \left.+\frac{2 \alpha \tilde{\tilde{\rho}}}{\tilde{\rho}}\left(f(\tilde{\rho})+2 \dot{\tilde{\rho}}^{2}+k+\frac{\tilde{\rho}^{2}}{4 \alpha}\right)\right]\left.\right|_{-} ^{+},
\end{aligned}
$$

where the dots stand for derivatives with respect to $\tau$. For a static timelike shell at $r=\rho$, such that $\dot{\tilde{\rho}}=0$ and $\ddot{\tilde{\rho}}=0$, we obtain

\footnotetext{
${ }^{5}$ A static throat with null or positive energy density (and $\alpha>0$ ) is incompatible with bulk regions of the $\xi=-1$ branch at both sides of the hypersurface [32].
} 


$$
\begin{aligned}
S_{\tau}^{\tau}= & \frac{\alpha}{2 \pi \rho^{3}}\left(\eta_{+} \sqrt{f_{+}(\rho)}-\eta_{-} \sqrt{f_{-}(\rho)}\right) \\
& \times\left[3\left(k+\frac{\rho^{2}}{4 \alpha}\right)-f_{+}(\rho)-f_{-}(\rho)-\eta_{+} \eta_{-} \sqrt{f_{+}(\rho) f_{-}(\rho)}\right],
\end{aligned}
$$

$$
\begin{aligned}
S_{\theta}^{\theta} & =S_{\phi}^{\phi}=S_{\chi}^{\chi} \\
& =\left(\frac{\eta_{+}}{\sqrt{f_{+}(\rho)}}-\frac{\eta_{-}}{\sqrt{f_{-}(\rho)}}\right)\left[k-\zeta(\rho)-\sqrt{f_{+}(\rho) f_{-}(\rho)} \eta_{+} \eta_{-}\right],
\end{aligned}
$$

where we used the definition

$$
\begin{aligned}
\zeta(\rho) & \equiv-\frac{2 \alpha}{\rho}\left(k+\frac{\rho^{2}}{4 \alpha}-f(\rho)\right) f^{\prime}(\rho)+k-f(\rho) \\
& =\frac{Q^{2}}{3 \rho^{4}}+\frac{\rho^{2} \Lambda}{3},
\end{aligned}
$$

and in the last equality of (12) it is assumed that $Q^{2}$ and $\Lambda$ are the same at both sides of the hypersurface. We recall that, despite that we are mainly interested in $\Lambda=0$ and $k=1$, we keep track of cosmological constant solutions and curvature parameter $k$ in some general expressions to be able to compare cases, as well as for comments on Sec. V.

We first consider vanishing stress tensor components in the static case to apply them to the construction of the throat. The inner regions of our wormhole have metric functions $f_{i}(a)$ at the position of the throat, at $r_{L}=r_{R}=a$, and the orientation factor for each bulk region at the throat is given by $\eta_{L i}=-1$ and $\eta_{R i}=1$. Putting all together in (11) and (12) the following two equations are obtained:

$$
\begin{aligned}
& f_{i}(a)=3\left(k+\frac{a^{2}}{4 \alpha}\right), \\
& f_{i}(a)=\zeta(a)-k .
\end{aligned}
$$

On the other hand, at the left and right side bubbles placed at $r_{L}=b$ and $r_{R}=b$, the exterior regions have metric functions $f_{e}(b)$, while the left and right interior regions have $f_{i}(b)$. The orientation factor for the bulk regions at each bubble is given by $\eta_{L i}=-1$ and $\eta_{L e}=-1$ for the left side bubble, and $\eta_{\operatorname{Re}}=1$ and $\eta_{R i}=1$ for the right side bubble. By demanding a vanishing stress tensor, the following two equations are obtained:

$$
\begin{aligned}
& \sqrt{f_{e}(b)} \sqrt{f_{i}(b)}=3\left(k+\frac{b^{2}}{4 \alpha}\right)-f_{e}(b)-f_{i}(b), \\
& \sqrt{f_{e}(b)} \sqrt{f_{i}(b)}=k-\zeta(b) .
\end{aligned}
$$

The latter four equations determine the possible configurations for the wormhole spacetime. A priori, from the simultaneous requirements in (15) and (17), we see that there are no solutions with a vanishing charge and a vanishing cosmological constant. Considering a nonvanishing charge only, $k=-1$ and $k=0$ curvatures are not admissible either. The remaining possibility with $\Lambda=0$ and $Q \neq 0$ is the spherical $(k=1)$ wormholes which are shown to exist and are studied below. The inclusion of $\Lambda \neq 0$ generates a variety of possibilities commented in the last section.

Now, let us study the space of solutions. To analyze the wormhole construction we use the following definitions for the configuration parameters:

$$
\begin{aligned}
x & \equiv \frac{a^{2}}{4 \alpha}, \quad y \equiv \frac{b^{2}}{4 \alpha}, \\
m_{i} & \equiv \frac{M_{i}}{\alpha}, \quad m \equiv \frac{M}{\alpha}, \\
q^{2} & \equiv \frac{1}{3}\left(\frac{Q}{4 \alpha}\right)^{2}, \quad \lambda \equiv \frac{4 \alpha \Lambda}{3} .
\end{aligned}
$$

Squaring conveniently Eq. (14), and combining it with (15), the static equations for a general symmetric vacuum throat are

$$
\begin{array}{r}
4 k x^{2}+\left(4 k^{2}-m_{i}\right) x+3 q^{2}=0, \\
3(3-\lambda) x^{2}+16 k x+\left(4 k^{2}-m_{i}\right)=0,
\end{array}
$$

with the condition $x+k>0$, not to lose the information of the sign in the original unsquared equation. Additionally, the requirement $x>x_{s}$, where $x_{s}=\left(r_{S_{i}}\right)^{2} /(4 \alpha)$ is the branch singularity corresponding to the interior radial coordinate $r_{S_{i}}$, must be considered. The latter equations establish the relation among $x, m_{i}, q$, and $\lambda$ as compatible with a generic symmetric vacuum throat.

It is worth pointing out that the existence of the wormhole solutions demands the presence of the highercurvature terms. This is why the GR limit $\alpha \rightarrow 0$ in the equations above does not yield any configuration of this class. Both finite values of the higher-curvature coupling $\alpha$ and the electric charge $Q$ are necessary for having solutions like the ones we presented here. Nevertheless, the role played by the higher-curvature terms and by the charge are different: while the former are needed and are sufficient to have wormhole solutions in five-dimensional EGB theory [46], the latter is what, as we will see below, suffices to render the solution stable under radial perturbations; cf. [32].

On the other hand, the vacuum bubbles at radial coordinates $b$ are constructed from the junction of an interior metric with $f_{i}(b)=k+y\left(1+I_{y}\right)$ and an exterior $f_{e}(b)=$ $k+y\left(1-E_{y}\right), \quad$ where $\quad I_{y} \equiv\left(1+\frac{m_{i}}{y^{2}}-\frac{2 q^{2}}{y^{3}}+\lambda\right)^{1 / 2} \quad$ and 
$E_{y} \equiv\left(1+\frac{m}{y^{2}}-\frac{2 q^{2}}{y^{3}}+\lambda\right)^{1 / 2}$, respectively. From Eqs. (16) and

(17) of the shell we read the general conditions $k+y>0$ and $k>\zeta_{y}$, with $\zeta_{y} \equiv \zeta(b)=(q / y)^{2}+y \lambda$. Additionally, after some manipulations, we have the inequalities $I_{y}>$ $E_{y}>I_{y} / 2$. Considering these conditions, the combined vacuum bubble equations can be expressed as

$$
\begin{gathered}
y I_{y} E_{y}=\left[3(k+y)-y\left(I_{y}-E_{y}\right)\right]\left(I_{y}-E_{y}\right), \\
\zeta_{y}+y=y\left(I_{y}-E_{y}\right) .
\end{gathered}
$$

We stress that $y>y_{h}=r_{H}^{2} /(4 \alpha)$ if the charge is lower than the critical value for the exterior metric, or if not, then $y>y_{s}=\left(r_{S_{e}}\right)^{2} /(4 \alpha)$ to avoid branch singularity. The latter equations and conditions give the relations among $y, m_{i}, m$, $q$, and $\lambda$ compatible with the static vacuum bubble. Note that the positivity of each side in (24) is fixed by the aforementioned conditions and, therefore, $M<M_{i}$ for the construction of the bubbles.

We are mainly interested in the spherical $k=1$ solutions with $\lambda=0$; the static throat configurations in this case are described by

$$
\begin{aligned}
& q^{2}=4 x^{2}+3 x^{3}, \\
& m_{i}=4+16 x+9 x^{2} .
\end{aligned}
$$

Evaluating with $\lambda=0$ and $k=1$, the condition $k>\zeta_{y}=$ $(q / y)^{2}$ becomes an inequality for charge and bubble radii which reads

$$
|q|<y
$$

and Eqs. (23) and (24) give $m_{i}$ and $m$ in terms of $q$ and $y$,

$$
\begin{aligned}
m_{i}= & \frac{1}{2}\left(6 y+3 y^{2}+(6+8 y) \frac{q^{2}}{y^{2}}-\frac{q^{4}}{y^{4}}\right. \\
& \left.+\left[3\left(4+3 y-\frac{q^{2}}{y^{2}}\right)\left(y+\frac{q^{2}}{y^{2}}\right)^{3}\right]^{1 / 2}\right), \\
m= & \frac{1}{2}\left(6 y+3 y^{2}+(6+8 y) \frac{q^{2}}{y^{2}}-\frac{q^{4}}{y^{4}}\right. \\
& \left.-\left[3\left(4+3 y-\frac{q^{2}}{y^{2}}\right)\left(y+\frac{q^{2}}{y^{2}}\right)^{3}\right]^{1 / 2}\right) .
\end{aligned}
$$

Combining the latter functions with those obtained for $m_{i}$ and $q^{2}$ in terms of $x$, from the shell in the throat, (25) and (26), we establish our vacuum wormhole solutions in parameter space. The compatible configurations are shown in the figures presented in the next section, with the corresponding stability analysis.

Small wormholes with $\lambda=0$ can be studied assuming the existence of configurations with $x \lesssim y \ll 1$. From (25) and (26), the interior mass and charge in this approximation are $m_{i} \simeq 4+16 x$ and $q^{2} \simeq 4 x^{2}$. Under this consideration $\zeta_{y}=(q / y)^{2} \simeq(2 x / y)^{2}$. Besides, considering the inequality $E_{y}>I_{y} / 2$, we have

$m>1+4 x+6 x^{2} / y+\frac{3}{2}\left(3 x^{2} / 2-y^{2} / 2+3 x^{3} / y\right)$,

for the exterior mass. Using the latter and Eqs. (28) and (29) to express the sum $m_{i}+m$ to first order in the small parameters, we have

$$
-\zeta_{y}^{2}+6 \zeta_{y}+y \theta \gtrsim 5
$$

where $\theta=\frac{13}{2} \zeta_{y}-10 \zeta_{y}{ }^{1 / 2}+6 \sim \mathcal{O}(1)$, and positive, for $0<\zeta_{y}<1$. Defining $\epsilon$ as a positive quantity of the same order as $x, y$, and $q$ in the small wormhole, the inequality (31) establishes that $1>\zeta_{y}{ }^{1 / 2}>1-\epsilon$, which is consistent with the assumptions and conditions. Finally, under this approximation,

$$
2 x \simeq y \zeta_{y}{ }^{1 / 2}=y-\mathcal{O}\left(\epsilon^{2}\right)
$$

and $q^{2}=y^{2}-\mathcal{O}\left(\epsilon^{3}\right)$. Using the latter to evaluate (29) we obtain

$$
m=1+4|q|-\mathcal{O}\left(\epsilon^{2}\right) .
$$

The charge is $|q|=\frac{m-1}{4}+\mathcal{O}\left(\epsilon^{2}\right)$ and, in terms of the original parameters of the metric, a small wormhole solution is compatible with a charge greater but approximately equal to the critical value, $|Q| / \alpha \simeq \sqrt{3}(M / \alpha-1)$, of the external black hole geometry. These solutions are shown in the small parameter regions of the figures in the next section, i.e., small dimensionless radii of the shells, $a / \sqrt{\alpha}$ and $b / \sqrt{\alpha}$, in Figs. 2(a) and 2(b), and small dimensionless charge $|Q| / \alpha$ in Fig. 3(a).

\section{STABILITY ANALYSIS}

The dynamics of throat and bubbles is determined from the junction conditions by analyzing the radii $a(\tau)$ and $b(\tau)$ as dynamical variables, introducing small perturbations around the equilibrium. We will consider the method originally introduced in [47] to study the stability of thin shells in GR. This amounts to finding an effective potential for the variables $a(\tau)$ and $b(\tau)$ and then studying the conditions for its convexity. This method of studying the thin shells "bounded excursion" has been considered extensively in the GR literature-see for instance Sec. 4.1 of [48] —and in the context of wormholes it has been originally considered in [44]. Here, we will first consider the stability of the throat alone, and then we add the analysis for the bubble to determine the stability of the complete configuration. 


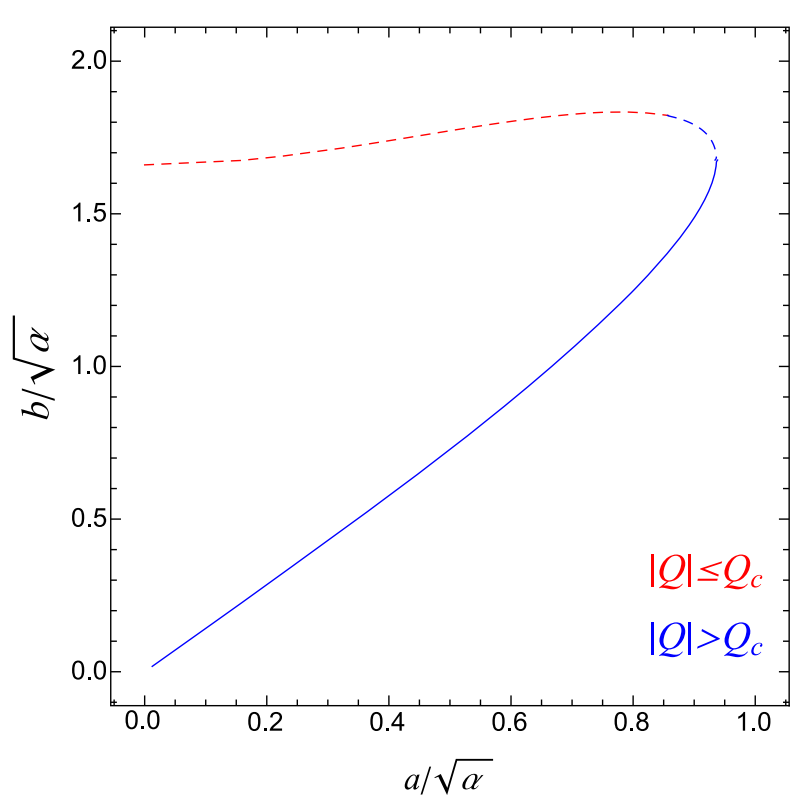

(a)

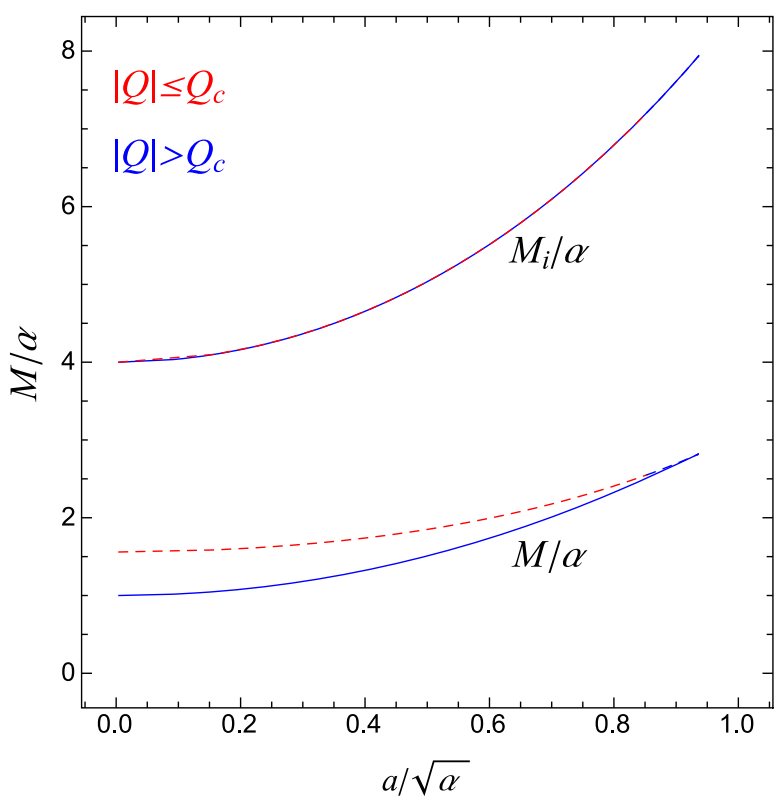

(b)

FIG. 2. Solid curves represent stable configurations (only possible with supercritical charge values). Dashed curves are unstable solutions. (a) Bubble radius against throat radius. (b) Interior mass (upper curve) and exterior mass (lower curve).

The dynamics of the throat at radius $\tilde{a} \equiv a(\tau)$ is described by $\dot{\tilde{a}}^{2}+V(\tilde{a})=0$, with the effective potential

$$
V(\tilde{a})=k+\tilde{x}-\tilde{x} \frac{\tilde{I_{x}}}{2}
$$

which follows from $S_{\tau}^{\tau}=0$ in (9), where we used $\tilde{x} \equiv \frac{\alpha^{2}(\tau)}{4 \alpha}$, and $\tilde{I}_{x} \equiv \sqrt{1+\frac{m_{i}}{\tilde{x}^{2}}-\frac{2 q^{2}}{\tilde{x}^{3}}+\lambda}$. The first derivative of the

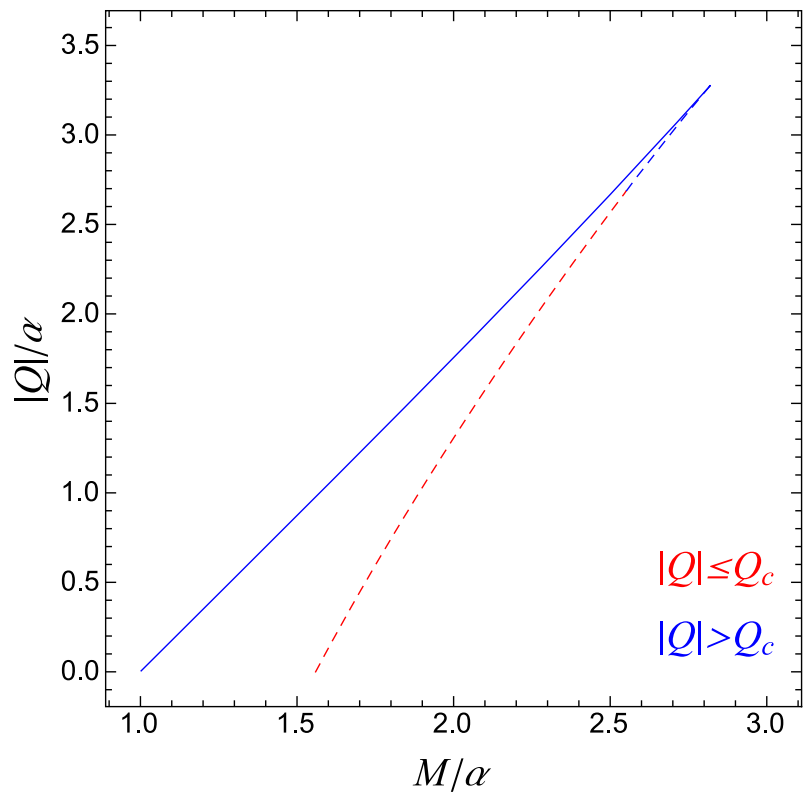

(a) potential evaluated at the static radius $a$ is $V^{\prime}(a)=0$, while for the second derivative we obtain

$$
V^{\prime \prime}(a)=\frac{3 q^{2}-4 k x^{2}}{4 \alpha x^{2}(k+x)},
$$

where the prime stands for the derivative with respect to the radius of the shell. Evaluating the latter for the wormhole

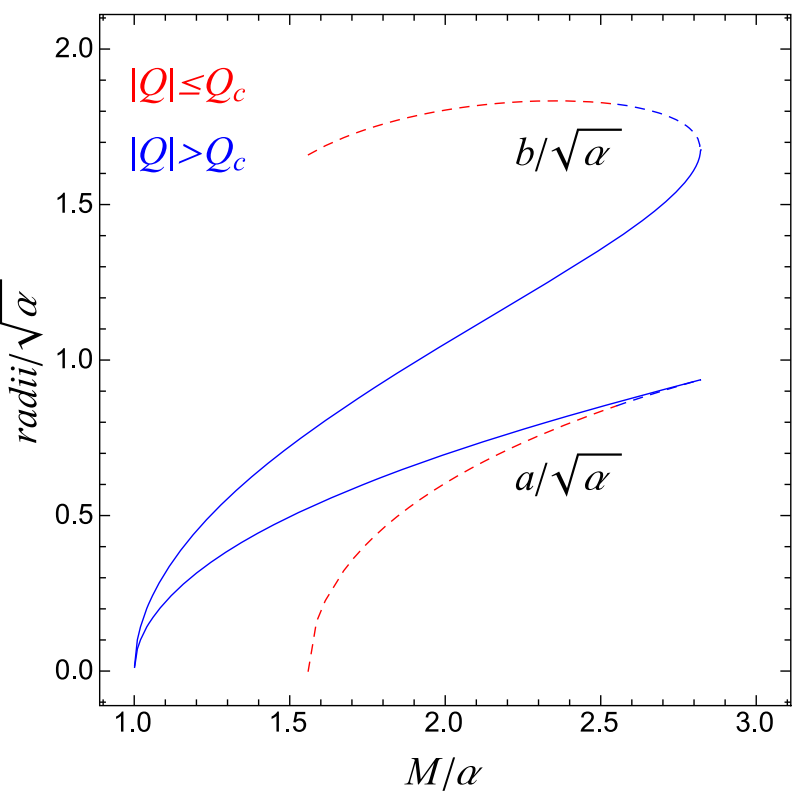

(b)

FIG. 3. Solid curves represent stable configurations (only possible with supercritical charge values). Dashed curves are unstable solutions. (a) Charge against external mass. (b) Bubble radii (upper curve) and throat radius (lower curve). 
configurations, with parameters given as in (25) and (26) in the throat, the second derivative of the potential at the equilibrium position is

$V^{\prime \prime}(a)=\frac{8+9 x}{4 \alpha(1+x)}>0, \quad q \neq 0, \lambda=0, k=1$,

yielding stable symmetric vacuum throats in every case; cf. [40]. The complete stability of shells in the wormhole we are interested in will exclusively depend on stability of the bubbles. ${ }^{6}$

Dynamics of the bubbles with radius $\tilde{b} \equiv b(\tau)$ is described by $\dot{\tilde{b}}^{2}+V(\tilde{b})=0$, with the effective potential

$$
V(\tilde{b})=k+\tilde{y}-\frac{\tilde{y}}{3}\left(\tilde{I}_{y}-\tilde{E}_{y}+\frac{\tilde{I}_{y} \tilde{E}_{y}}{\tilde{I}_{y}-\tilde{E}_{y}}\right)
$$

where the dynamical parameter is $\tilde{y} \equiv \frac{b^{2}(\tau)}{4 \alpha}$. Evaluating at the static position of radius $b$ we have $\tilde{y}=y, \tilde{I}_{y}=I_{y}$, and $\tilde{E}_{y}=E_{y}$. The first derivative of the potential at the equilibrium position is $V^{\prime}(b)=0$, while for the second derivative we obtain

$$
V^{\prime \prime}(b)=\frac{1}{\alpha}\left(\frac{3 q^{2} / y^{3}}{I_{y}-E_{y}}-\frac{I_{y}-E_{y}}{I_{y} E_{y}}-1\right) .
$$

The stability requirement $V^{\prime \prime}(b)>0$ can be expressed generically as

$9 q^{2}(k+y)-\left(\zeta_{y}+y\right)\left[3 q^{2}+y^{2}\left(3(k+y)-\zeta_{y}\right)\right]>0$,

where we used Eqs. (23) and (24), together with the general condition $\zeta_{y}+y>0$ for vacuum static bubbles. From the latter, a general feature can be mentioned; bubbles are unstable without electromagnetic field for any possible value of the cosmological constant. Then, only charged configurations would admit stable vacuum bubbles.

The complete stability of the shells in our wormhole is therefore obtained from the latter inequality with $k=1$ and $\zeta_{y}=q^{2} / y^{2}$. The space of solutions for the electrovacuum wormhole spacetime with flat asymptotics, together with the stability condition, are shown in the following figures. Figure 2 shows the dimensionless bubble radius and

\footnotetext{
${ }^{6}$ For completeness, we mention here that, including cosmological constant solutions, the general symmetric throat has $\lambda=3+4 k / x-q^{2} / x^{3}, m_{i}=4 k^{2}+4 k x+3 q^{2} / x$ and is stable if $3 q^{2}>4 k x^{2}$, as it is given by (35) by using the general condition $k+x>0$. We note that if there were a vanishing charge, the stability would depend exclusively on the value of the curvature $k$. Particularly, if $q=0$ and $k=1$ solutions exist only for $\lambda>0$ and are unstable under radial perturbations.
}

dimensionless mass parameters against dimensionless throat radius $a / \sqrt{\alpha}$. Figure 3 shows the dimensionless charge and shells radii against dimensionless external mass $M / \alpha$. The curves in the different sets of parameter axes represent the wormhole solutions. Solid line curves represent stable configurations, and dashed lines are unstable. Stable configurations occur only with charges greater than the critical value given by the external asymptotically flat black hole metric, the cases with $|Q|>Q_{c}$ are painted in blue, while $|Q| \leq Q_{c}$ are in red.

The neutral equilibrium condition $V^{\prime \prime}(b)=0$ for the configuration space of the bubble can be obtained by equating to zero the left-hand side of (39) with $k=1$ and $\lambda=0$. This gives the relation between $y$ and the dimensionless charge $q$ in the configuration of neutral equilibrium of the bubble, namely

$$
q_{n e}^{2}=\frac{y_{n e}^{2}}{2}\left(3+2 y_{n e}-\sqrt{9+6 y_{n e}-2 y_{n e}^{2}}\right) .
$$

By introducing this charge in the formulas for the static configurations (28) and (29) we would obtain the mass parameters for the neutral equilibrium curves of the bubble; these are $m=m\left(y_{n e}\right)$ and $m_{i}=m_{i}\left(y_{n e}\right)$. On the other hand, considering Eqs. (25) and (26) for the static throat of our wormhole we can write the charge in terms of interior mass as

$$
q^{2}=\frac{1}{243}\left[\left(28+9 m_{i}\right)^{3 / 2}-80-108 m_{i}\right]
$$

for $m_{i}>4$. Requiring these two square charges to be the same, by evaluating the latter at $m_{i}=m_{i}\left(y_{n e}\right)$, we achieve the neutral equilibrium point in the wormhole space of solutions. Solving accordingly we obtain that $y_{n e} \simeq 0.70$ and, for the original metric parameters at the neutral equilibrium point, we have $a_{n e} \simeq 0.94 \sqrt{\alpha}$, $b_{n e} \simeq 1.68 \sqrt{\alpha},|Q|_{n e} \simeq 3.28 \alpha, M_{n e} \simeq 2.82 \alpha$, and $M_{i n e} \simeq$ $7.94 \alpha$. Each of these neutral equilibrium parameters can be read in the corresponding plotted figure. Stable wormholes occur with bubble radius $b<b_{n e}$ and charges greater than the critical charge of the exterior black hole manifolds. The corresponding mass and charge for these solutions ranges in $\alpha<M<M_{n e}$ and $0<|Q|<|Q|_{n e}$, respectively.

From the stability analysis, we conclude that the $U(1)$ charge $Q$ is what stabilizes the wormhole solution under small scalar perturbations; cf. [32]. It is worth pointing out that the stable solutions require supercritical values $|Q|>Q_{c}$. This might be relevant for some questions on quantum gravity: In the path integral approach, one formally defines the theory as the fluctuations about saddle points that obey certain asymptotic conditions that define charges at infinity. One picks such saddles from a set of physically sensible classical solutions, typically excluding 
naked singularities and other pathological configurations. This means that certain regions of the space of charges will in principle be excluded. For example, solutions (2) and (3) with $|Q|>Q_{c}$ are expected to develop a branch singularity, and therefore one excludes such values of the charge. Here, we are finding that completely regular solutionswith two asymptotic regions - actually exist for supercritical values of the charge, and thus such a bound should be reconsidered. Conversely, the analysis above also tells us that wormhole solutions with $|Q|<Q_{c}$ are unstable, and this means something about the uniqueness of the solutions (2) and (3) for a given set of variables $M$ and $|Q|<Q_{c}$.

\section{ANTI-DE SITTER SPACE}

Wormhole solutions also exist in (A)dS spacetime provided $\Lambda \neq 0$. Probably, the most interesting examples are the AdS wormholes, as they allow one to think of their implications for AdS/CFT. These geometries have two asymptotically $\mathrm{AdS}_{5}$ regions of effective cosmological constant

$$
\Lambda_{\mathrm{eff}}=\frac{3}{2 \alpha}(\sqrt{1+\lambda}-1)
$$

There are several questions one can ask about the interest of these solutions in the context of holography: First, one could ask whether the theory admits locally $\mathrm{AdS}_{5}$ solutions with flat base manifolds (i.e., $k=0$ ). These solutions correspond to the boundary being locally $\mathbb{R}^{1,3}$. Interestingly, the theory (1) with $\Lambda<0$ does admit ${ }^{7}$ both planar $(k=0)$ and hyperbolic $(k=-1)$ wormhole solutions. In these cases, condition (17) at the vacuum bubbles is only compatible for negative $\Lambda$, while charge $Q$ is, again, an essential ingredient to satisfy Eq. (15) in a planar wormhole throat. The locally AdS solution with $k=1$ is also possible; it requires the presence of a nonzero electromagnetic field, as it follows from (15).

A second question one can ask is whether the wormhole solutions are allowed by the so-called causality bounds. To answer this question one has to compare the range of the dimensionless parameter $\lambda$ where

\footnotetext{
${ }^{7}$ The case $k=+1$ is possible with both positive and negative $\Lambda$. The cases $k=0$ and $k=-1$ are only possible with $\Lambda<0$.
}

the stable $\mathrm{AdS}_{5}$ wormholes are possible with the causality segment ${ }^{8}$

$$
-\frac{9}{25}<\lambda<\frac{7}{9}
$$

that comes from $\mathrm{AdS}_{5} / \mathrm{CFT}_{4}$. It turns out that stable wormhole solutions can be seen to exist within the range $-1 \leq \lambda \lesssim-0.83$, which means that the causality bound excludes such solutions. An interesting particular case, which cannot be excluded by the standard causality arguments, is $\lambda=-1$. At this point of the parameter space, the theory can be written as five-dimensional $S O(4,2)$ Chern-Simons gauge theory, it has a unique maximally symmetric vacuum, and it also exhibits other special features. At this point, there are no local degrees of freedom around the vacuum [41], and this is why it cannot be excluded by the standard causality arguments. $\mathrm{AdS}_{5}$ wormholes at $\lambda=-1$ have previously been considered in the context of $\mathrm{AdS}_{5} / \mathrm{CFT}_{4}$, for instance, in Refs. [49,50]. Traversable $\mathrm{AdS}_{5}$ wormholes are dual to a pair of $\mathrm{CFT}_{4}$ 's interacting with each other. In [49,50], configurations in which a pair of charges is present in each copy of the $\mathrm{CFT}_{4}$ were considered. A phase transition is seen to occur when the particles of each pair are separated from each other. A similar phenomenon is expected to occur in the wormhole geometries we constructed here, although there are some differences: Appropriate boundary conditions for the string configuration have to be considered in the nondifferentiable junctions $\Sigma_{L, R}^{b}$ and $\Sigma^{a}$, and the effect of the nonvanishing charge has to be taken into account. We plan to investigate the holographic interpretation of the wormhole solution at $\lambda=$ -1 in the future.

\section{ACKNOWLEDGMENTS}

The authors thank Mariano Chernicoff and Julio Oliva for discussions. This work has been supported by CONICET and University of Buenos Aires Facultad de Ciencias Exactas y Naturales-UBA.

\footnotetext{
${ }^{8}$ It is worth comparing with Ref. [19], where the cosmological constant is denoted $\Lambda=-6 / L^{2}$ and the coupling constant of the Gauss-Bonnet quadratic terms is denoted $\alpha=\lambda_{\mathrm{GB}} L^{2} / 2$. Here, we define the parameter $\lambda \equiv 4 \Lambda \alpha / 3$, i.e., $\lambda=-4 \lambda_{\mathrm{GB}}$. Therefore, the causality bounds on the Gauss-Bonnet coupling coming from $\mathrm{AdS}_{5} / \mathrm{CFT}_{4}$ reads as (43); see for instance the bound after Eq. (4.9) of [19], which translates into the lower bound in (43). In the notation of [19], the Chern-Simons point $\lambda=-1$ reads $\lambda_{\mathrm{GB}}=1 / 4$.
} 
[1] C. W. Misner and J. A. Wheeler, Classical physics as geometry: Gravitation, electromagnetism, unquantized charge, and mass as properties of curved empty space, Ann. Phys. (N.Y.) 2, 525 (1957).

[2] M. S. Morris and K. S. Thorne, Wormholes in space-time and their use for interstellar travel: A tool for teaching general relativity, Am. J. Phys. 56, 395 (1988).

[3] R. W. Fuller and J.A. Wheeler, Causality and multiply connected space-time, Phys. Rev. 128, 919 (1962).

[4] J. Maldacena and L. Susskind, Cool horizons for entangled black holes, Fortschr. Phys. 61, 781 (2013).

[5] M. Chernicoff, A. Güijosa, and J. F. Pedraza, Holographic EPR pairs, wormholes and radiation, J. High Energy Phys. 10 (2013) 211.

[6] P. Gao, D. L. Jafferis, and A. Wall, Traversable wormholes via a double trace deformation, J. High Energy Phys. 12 (2017) 151.

[7] J. Maldacena, D. Stanford, and Z. Yang, Diving into traversable wormholes, Fortschr. Phys. 65, 1700034 (2017).

[8] J. Maldacena and X. L. Qi, Eternal traversable wormhole, arXiv:1804.00491.

[9] P. Gao and H. Liu, Regenesis and quantum traversable wormholes, arXiv:1810.01444.

[10] E. Caceres, A. S. Misobuchi, and M. L. Xiao, Rotating traversable wormholes in AdS, J. High Energy Phys. 12 (2018) 005.

[11] P. Betzios, E. Kiritsis, and O. Papadoulaki, Euclidean wormholes and holography, J. High Energy Phys. 06 (2019) 042.

[12] B. Freivogel, V. Godet, E. Morvan, J. F. Pedraza, and A. Rotundo, Lessons on eternal traversable wormholes in AdS, arXiv:1903.05732.

[13] J. Maldacena, A. Milekhin, and F. Popov, Traversable wormholes in four dimensions, arXiv:1807.04726.

[14] E. Ayon-Beato, F. Canfora, and J. Zanelli, Analytic selfgravitating Skyrmions, cosmological bounces and AdS wormholes, Phys. Lett. B 752, 201 (2016).

[15] A. Anabalón and J. Oliva, Four-dimensional traversable wormholes and bouncing cosmologies in vacuum, J. High Energy Phys. 04 (2019) 106.

[16] A. Anabalón, B. de Wit, and J. Oliva, Supersymmetric traversable wormholes in four space-time dimensions, to be published.

[17] G. T. Horowitz, D. Marolf, J. E. Santos, and D. Wang, Creating a traversable wormhole, arXiv:1904.02187.

[18] N. Graham and K. D. Olum, Achronal averaged null energy condition, Phys. Rev. D 76, 064001 (2007).

[19] M. Brigante, H. Liu, R. C. Myers, S. Shenker, and S. Yaida, Viscosity bound violation in higher derivative gravity, Phys. Rev. D 77, 126006 (2008).

[20] D. M. Hofman, Higher derivative gravity, causality and positivity of energy in a UV complete QFT, Nucl. Phys. B823, 174 (2009).

[21] I. Antoniadis, S. Ferrara, R. Minasian, and K. S. Narain, $\mathrm{R} * * 4$ couplings in $\mathrm{M}$ and type II theories on Calabi-Yau spaces, Nucl. Phys. B507, 571 (1997).

[22] S. Ferrara, R. R. Khuri, and R. Minasian, M theory on a Calabi-Yau manifold, Phys. Lett. B 375, 81 (1996).
[23] M. Guica, L. Huang, W. Li, and A. Strominger, R**2 corrections for 5-D black holes and rings, J. High Energy Phys. 10 (2006) 036.

[24] C. Lanczos, A remarkable property of the RiemannChristoffel tensor in four dimensions, Ann. Math. 39, 842 (1938).

[25] D. Lovelock, The four-dimensionality of space and the Einstein tensor, J. Math. Phys. (N.Y.) 13, 874 (1972).

[26] B. Zwiebach, Curvature squared terms and string theories, Phys. Lett. 156B, 315 (1985).

[27] X. O. Camanho, J. D. Edelstein, J. Maldacena, and A. Zhiboedov, Causality constraints on corrections to the graviton three-point coupling, J. High Energy Phys. 02 (2016) 020.

[28] X. O. Camanho, J.D. Edelstein, G. Giribet, and A. Gomberoff, A new type of phase transition in gravitational theories, Phys. Rev. D 86, 124048 (2012).

[29] K. Izumi, Causal structures in Gauss-Bonnet gravity, Phys. Rev. D 90, 044037 (2014).

[30] H. Reall, N. Tanahashi, and B. Way, Causality and hyperbolicity of Lovelock theories, Classical Quantum Gravity 31, 205005 (2014).

[31] D. Lovelock, The Einstein tensor and its generalizations, J. Math. Phys. (N.Y.) 12, 498 (1971).

[32] C. Garraffo, G. Giribet, E. Gravanis, and S. Willison, Gravitational solitons and $\mathrm{C} 0$ vacuum metrics in fivedimensional Lovelock gravity, J. Math. Phys. (N.Y.) 49, 042502 (2008).

[33] M. G. Richarte and C. Simeone, Thin-shell wormholes supported by ordinary matter in Einstein-Gauss-Bonnet gravity, Phys. Rev. D 76, 087502 (2007); Erratum, Phys. Rev. D 77, 089903(E) (2008).

[34] C. Simeone, Addendum to 'Thin-shell wormholes supported by ordinary matter in Einstein-Gauss-Bonnet gravity', Phys. Rev. D 83, 087503 (2011).

[35] A. Belin, D. M. Hofman, and G. Mathys, Einstein gravity from ANEC correlators, arXiv:1904.05892.

[36] G. Dotti, J. Oliva, and R. Troncoso, Static wormhole solution for higher-dimensional gravity in vacuum, Phys. Rev. D 75, 024002 (2007).

[37] G. Dotti, J. Oliva, and R. Troncoso, Exact solutions for the Einstein-Gauss-Bonnet theory in five dimensions: Black holes, wormholes and spacetime horns, Phys. Rev. D 76, 064038 (2007).

[38] D. H. Correa, J. Oliva, and R. Troncoso, Stability of asymptotically AdS wormholes in vacuum against scalar field perturbations, J. High Energy Phys. 08 (2008) 081.

[39] H. Maeda and M. Nozawa, Static and symmetric wormholes respecting energy conditions in Einstein-Gauss-Bonnet gravity, Phys. Rev. D 78, 024005 (2008).

[40] T. Kokubu, H. Maeda, and T. Harada, Does the GaussBonnet term stabilize wormholes?, Classical Quantum Gravity 32, 235021 (2015).

[41] J. Zanelli, Lecture Notes on Chern-Simons (Super-) Gravities, 2nd ed. (2008).

[42] D. G. Boulware and S. Deser, String Generated Gravity Models, Phys. Rev. Lett. 55, 2656 (1985).

[43] D. L. Wiltshire, Black holes in string generated gravity models, Phys. Rev. D 38, 2445 (1988). 
[44] E. Poisson and M. Visser, Thin shell wormholes: Linearization stability, Phys. Rev. D 52, 7318 (1995).

[45] S. C. Davis, Generalized Israel junction conditions for a Gauss-Bonnet brane world, Phys. Rev. D 67, 024030 (2003).

[46] C. Garraffo and G. Giribet, The Lovelock black holes, Mod. Phys. Lett. A 23, 1801 (2008).

[47] P. R. Brady, J. Louko, and E. Poisson, Stability of a shell around a black hole, Phys. Rev. D 44, 1891 (1991).
[48] M. Visser and D. L. Wiltshire, Stable gravastars: An alternative to black holes?, Classical Quantum Gravity 21, 1135 (2004).

[49] M. Ali, F. Ruiz, C. Saint-Victor, and J. F. VazquezPoritz, Strings on AdS wormholes, Phys. Rev. D 80, 046002 (2009).

[50] M. Ali, F. Ruiz, C. Saint-Victor, and J. F. Vazquez-Poritz, Strings on AdS wormholes, J. Phys. Conf. Ser. 462, 012058 (2013). 